\title{
Typology-Based Analysis of Covid-19 Mobile Applications: Implications for Patient Empowerment
}

\author{
Riikka VUOKKO ${ }^{\mathrm{a}, 1}$, Kaija SARANTO ${ }^{\mathrm{b}}$ and Sari PALOJOKI ${ }^{\mathrm{a}}$ \\ ${ }^{\mathrm{a}}$ The Ministry of Social Affairs and Health, Finland \\ ${ }^{\mathrm{b}}$ University of Eastern Finland, Finland
}

\begin{abstract}
During COVID-19 pandemic, mobile technology is seen as potential tool for epidemic control and citizens' empowerment. Based on literature, we explore, which are the currently known types of the mobile apps and what implications do the apps have for patient empowerment. There is a need for evidence and an assessment framework to ensure that COVID-19 apps deliver on their promises.
\end{abstract}

Keywords. COVID-19, mobile application, tracing, remote technologies, empowerment

\section{Introduction}

During COVID-19 pandemic, mobile technology is being envisioned as potential and ubiquitous tool for authorities' epidemic control. At the same time, mobile technology has potential to provide easily accessible information for the citizens. Targeting those goals, COVID-19-related smartphone, and web-based health applications (later apps) are being rapidly developed, leading to a multitude of options, raising ethical and legal challenges and potentially confusing end users. [1,2]

The increasing presence of technology in health care has created new opportunities for patient engagement and with this, an emerging exploration of patient empowerment within the digital health context. Research gives evidence that there is a linkage between digital health solutions, and patient empowerment, but measurable health outcomes remains yet elusive [3]. Alarmingly, there is currently a lack of real-world evidence for potentially beneficial mobile applications used by citizens and patients during the COVID-19 pandemic for their need of information and support for coping. Health literacy and - in this context - the digital divide are important aspects of empowerment but remaining challenges in this are less discussed even though they may hinder maximizing the potential of mobile tools $[4,5]$.

Due to a diversity of COVID-19 apps with abundant objectives, it is important to support professionals and the public in identifying the varied types and functionalities of the apps. Additionally, taken that apps promote health-care intervention it is substantive to outline their impact on patient empowerment. Therefore, our research

1 Corresponding author, Dr. Riikka Vuokko, P.O. Box 33, 00023 Helsinki, Finland, riikka.vuokko@gmail.com. 
questions are: (1) What are the functionalities of currently known types of COVID-19 mobile apps? (2) What implications do the apps have in regard of patient empowerment?

\section{Methods}

In this paper, we apply approach of typologies, similar to classifications as useful tools to classify and organise items based on common variables (attribute such as colour), where the types are mutually exclusive (e.g., red type) and the typology system complete, although in the real world, people tend to disagree of their nature. [6, 7] European Commission (later EC) identifies four types of COVID-19 applications based on their services: symptom checkers and self-diagnosis apps, apps for tracking the spread of the coronavirus, apps for delivering trustworthy information and guidelines to public, and apps for supporting homebound patients and enabling selfmanagement. [2] Alternative typology is suggested based on the outcomes of the apps: whether their goal is in societal impact, in personal impact or in density dependence [1]. While there is yet little evidence of the apps' outcomes, in this paper, we concentrate on the EC typology based exploration of the COVID-19 apps. Terms for literature searches were composed according to the typology: "Covid-19", "apps", "guideline", "information", "self-diagnosis", "symptom checker", "symptom", "tracking", "tracing", "home", "self-management", "triage", "coping".

In this context, we conceptualize patient empowerment to cover situations where citizens are encouraged to take an active role in the management of their own health [5, 9]. Patient empowerment is a meta-paradigm and it is a broader concept than patient participation and patient-centeredness. [8]

PubMed search in the middle of July 2020 resulted in 28 peer-reviewed papers. When the concept of empowerment was composed with other search terms, it did not result in added papers. After removing duplicates and the first exclusion round based on two researcher reading the abstracts (out of scope, e.g., focus on professionals, not relevant e.g., focus on dark net activities, language) 20 papers were selected for further reading. After full paper reading, additional five of the research papers were excluded as they were out of scope, or focused on professionals. Total of 15 papers were analysed using the EC typology.

\section{Results}

The first results based on PubMed searches indicate that there is yet little evidence for research of COVID-19 apps and that the terms describing these apps are not well established. Of the types, symptom checkers and self-diagnosis apps resulted in 3 papers, tracing apps 7, apps for information and guidelines 2, and monitoring apps 5 papers, when two papers covered several types of apps.

Results of the apps for symptom checking and triage show that evidence on these kinds of apps is scarce. While numerous apps are available for professionals, patients' perspective remains understudied. [10]. Devising personalized self-testing kits for COVID-19 virus is important because providing real-time testing will facilitate speedy prediagnosis to a large population [11]. Smartphone embedded software and highperformance computing have the potential to be deployed as self-test breathing 
monitoring apps. Those with higher risks of severe illness can check their breathing sound pattern frequently through the app [11]. Communication of health needs is of paramount importance when patients are isolated. Usage of alternative digital mental health options such as smartphone apps has increased, thus providing support for empowerment. The wide availability of these resources may promote resilience and well-being on a wider community level as mental health information is disseminated widely and potentially destigmatizes illness while promoting acceptance of digital tools. On turn, developing digital mental health resources without an evidence-based framework might be harmful. [12]

Results of the tracing apps give evidence of potentially useful tools that may be employed to limit disease transmission $[1,13]$. Several countries have now started to deploy apps capable of supporting COVID-19 contact tracing, but the efficacy of such apps has yet to be proved. Key functionalities include that apps inform people that they have spent a specific time near someone with the virus. The contacts should then respond according to local rules, for example by self-quarantining themselves immediately [1, 13-17]. These apps are not without concerns from a user perspective and consequently, they may cause limitations for patient empowerment. The topic of user adoption is presented align with privacy concerns, where some users may not be comfortable with an app that tracks their location or has otherwise negative effects on individual privacy. Users may become fatigued from procedures, e.g., scanning QR code, and choose to discontinue. $[13,17]$ False negative alarms could spur a false sense of safety in others. Moreover, many apps work only with certain phones causing uncertainties for availability. [15]. In turn, an emerging evidence suggests also the app may enable some patients to return more quickly to their lives [18]. In summary, literature evidences that these apps can contribute towards a more general, population level goal but a personal benefit and impact on empowerment is not as evident [1].

Results of the apps for information and guidance illustrate that during the pandemic people have a need for timely information and guidance when they seek for the latest news of the pandemic, check facts when encountering uncertainties, and want to obtain informational guidance for health management. [19] Typically information and guidance can be received autonomously, which supports citizens' selfdetermination and control, which are close coupled with empowerment. The information content in an app should be reliable and based on current data. [1]

Results of the apps for coping and monitoring at home emphasize necessity to avoid traditional face-to-face visits especially for patients with higher risks, such as elderly, without hampering the quality of care. During COVID-19 pandemic especially outpatient visits have been cancelled or postpone and digital technologies have become a way for accessing remote care. Advances in remote care and monitoring, e.g., via apps enable variety of possibilities for virtual visits, follow-ups, monitoring and consultation. [12, 20-23] At the same time, remote technologies, such as videoconferences, video monitoring and wearable devices, can provide electronic reminders and support in daily activities. Reported advantages of these kinds of apps are improved access and quality of care regardless of location or time, thus prompting full potential of empowerment. [12, 20-21] Reported limitations are technical issues, patients' and caregivers' skills with technology, and ethical concerns related to data privacy. [20] Consequently, while apps can increase agency in self-care and improvement in health, ability to share data captured with the devices back to caregivers remains a challenge, therefore limiting potential patient participation. [22] 


\section{Discussion}

Having applied a structured approach of a typology-based analysis, four types of COVID-19 apps and related functions were identified. Our results show that current development concentrates on two types of apps, namely the apps for tracing and for remote care and monitoring. Taken that the development of the apps has been exceptionally rapid due to pressure set by the ongoing pandemic some compromising ways in developing these apps have inevitably been applied. It should be noted that when developing apps, methods should be backed by scientific evidence. [1] An indepth analysis of comparison and consideration of the relative benefits and possible harms is required. [18] Structured assessment of already deployed apps is needed [1]. As most apps' use is still at initial stages, their full impact is yet unknown but scientific evidence and assessment would support recognizing their potential. This would illustrate which of the apps are effective and applicable for wider use which is a prerequisite for e.g., tracing apps $[1,12,15,16]$. To sum up our results, it is obvious that future evidence of COVID-19 digital interventions is urgently needed [19].

Plausible evidence of the types of apps and their implications to empowerment are yet scarce. Although empowerment is being articulated, structures emerging and supporting it are yet mostly unanalyzed. [9]. Research may give evidence how apps advance an emerging view of patient empowerment. Considering the nature of the pandemic as public health threat, we suggest exploring apps' impact on preventive behaviour and empowerment. [19] Especially, as a result of our analysis, the apps as ubiquitous technology supporting equity in care needs further evidence [18]. In the context of empowerment, it is critical to raise the fact that the introduction of new technologies can cause discrimination. This can take the form of bias where technology is available to some but not all. Thus, it is crucial to recognize the importance of equity when deploying apps if patient empowerment is one of the goals. $[1,18]$

Our approach is subject to some limitations. We wish to highlight a number of factors affecting reliability and validity of research, which deserve attention: the number of apps, the purpose of apps including a possible collection of functions, and an analysis framework for data should be clearly stated also in seminal research. Due to the ongoing situation, preliminary reporting is descriptive and may be selective or biased data. [23] While we applied EC typology for the current apps, different types of apps may dominate when the pandemic situation evolves. Moreover, no established frameworks or terminology is available for analyzing COVID-19 apps and patient empowerment, which is among the recognized development aims in the future.

To conclude, there is a need for evidence of apps' outcomes and their impact on empowerment. An assessment framework to evaluate how COVID-19 apps deliver on their promises should be established. Collaborative initiatives should harness both conventional and novel evidence-based tools to provide an effective and timely response to the COVID-19 pandemic on the global stage. [1, 2, 23]

\section{References}

[1] Vokinger KN, Nittas V, Witt CM, Fabrikant SI, von Wyl V. Digital health and the COVID-19 epidemic: an assessment framework for apps from an epidemiological and legal perspective. Swiss Med Wkly. 2020 May 17;150:w20282. doi: 10.4414/smw.2020.20282. PMID: 32418194. 
[2] European Commission. Mobile applications to support contact tracing in the EU's fight against COVID19. Common EU Toolbox for Member States. Version 1.0, 15.04.2020 https:/ec.europa.eu/health/sites/health/files/ehealth/docs/covid-19_apps_en.pdf.

[3] Risling T, Martinez J, Young J, Thorp-Froslie N. Defining Empowerment and Supporting Engagement Using Patient Views From the Citizen Health Information Portal: Qualitative Study JMIR Med Inform 2018;6(3):e43 URL: https://medinform.jmir.org/2018/3/e43 doi: 10.2196/medinform.8828 PMID: 30201603 PMCID: 6231721.

[4] Demiris G. Consumer Health Informatics: Past, Present, and Future of a Rapidly Evolving Domain. Yearb Med Inform. 2016 May 20;Suppl 1(Suppl 1):S42-7. doi: 10.15265/IYS-2016-s005. PMID: 27199196; PMCID: PMC5171509.

[5] Calvillo J, Román I, Roa LM. How technology is empowering patients? A literature review. Health Expect. 2015 Oct;18(5):643-52. doi: 10.1111/hex.12089. Epub 2013 May 28. PMID: 23711169; PMCID: PMC5060865.

[6] Chute CG. Clinical Classification and Terminology: Some History and Current Observations. J Am Med Inform Assoc. May-Jun 2000;7(3):298-303. doi: 10.1136/jamia.2000.0070298.

[7] Bowker GC, Star SL. Sorting Things Out: Classification and Its Consequences. $4^{\text {th }}$ ed. Cambridge, Mass: MIT Press; c1999. 377 p.

[8] Castro EM, Van Regenmortel T, Vanhaecht K, Sermeus W, Van Hecke A. Patient empowerment, patient participation and patient-centeredness in hospital care: A concept analysis based on a literature review. Patient Educ Couns. 2016 Dec;99(12):1923-1939. doi: 10.1016/j.pec.2016.07.026. Epub 2016 Jul 18. PMID: 27450481.

[9] Umar A, Mundy D. Re-thinking Models of Patient Empowerment. Stud Health Technol Inform. 2015;209:175-81. PMID: 25980722.

[10] Cervino G, Oteri G. COVID-19 Pandemic and Telephone Triage before Attending Medical Office: Problem or Opportunity? Medicina (Kaunas). 2020 May 20;56(5):250. doi: 10.3390/medicina56050250. PMID: 32443828; PMCID: PMC7279364.

[11] Faezipour M, Abuzneid A. Smartphone-Based Self-Testing of COVID-19 Using Breathing Sounds. Telemed J E Health. 2020 Jun 2. doi: 10.1089/tmj.2020.0114. Epub ahead of print. PMID: 32487005.

[12] Marshall JM, Dunstan DA, Bartik W. The role of digital mental health resources to treat trauma symptoms in Australia during COVID-19. Psychol Trauma. 2020 Jun 4. doi: 10.1037/tra0000627. Epub ahead of print. PMID: 32496103.

[13] Yasaka TM, Lehrich BM, Sahyouni R. Peer-to-Peer Contact Tracing: Development of a PrivacyPreserving Smartphone App. JMIR Mhealth Uhealth. 2020 Apr 7;8(4):e18936. doi: 10.2196/18936. PMID: 32240973; PMCID: PMC7144575.

[14] Morley J, Cowls J, Taddeo M, Floridi L. Ethical guidelines for COVID-19 tracing apps. Nature. 2020 Jun;582(7810):29-31. doi: 10.1038/d41586-020-01578-0. PMID: 32467596.

[15] Editorial: Show evidence that apps for COVID-19 contact-tracing are secure and effective. Nature. 2020 Apr;580(7805):563. doi: 10.1038/d41586-020-01264-1. PMID: 32350479.

[16] Zastrow M. Coronavirus contact-tracing apps: can they slow the spread of COVID-19? Nature. 2020 May 19. doi: 10.1038/d41586-020-01514-2. Epub ahead of print. PMID: 32433633.

[17] Bengio Y, Ippolito D, Janda R, Jarvie M, Prud'homme B, Rousseau JF, Sharma A, Yu YW. Inherent privacy limitations of decentralized contact tracing apps. J Am Med Inform Assoc. 2020 Jun 25:ocaa153. doi: 10.1093/jamia/ocaa153. Epub ahead of print. PMID: 32584990.

[18] Parker MJ, Fraser C, Abeler-Dörner L, Bonsall D. Ethics of instantaneous contact tracing using mobile phone apps in the control of the COVID-19 pandemic. J Med Ethics. 2020 Jul;46(7):427-431. doi: 10.1136/medethics-2020-106314. Epub 2020 May 4. PMID: 32366705; PMCID: PMC7231546.

[19] Liu PL. COVID-19 Information Seeking on Digital Media and Preventive Behaviors: The Me-diation Role of Worry. Cyberpsychology, Behavior, and Social Networking. 2020 Jun. https://doi.org/10.1089/cyber.2020.0250. Online ahead of Print.

[20] Cuffaro L, Di Lorenzo F, Bonavita S, Tedeschi G, Leanica L, Lavargna L. Dementia care and COVID19 pandemic: a necessary digital revolution. Neurological Sciences Jun 2020 41(8): 1977-1979. doi: 10.1007/s10072-020-04512-4.

[21] Greiwe J, Nyeanhuis SM. Wearable Technology and How This Can Be Implemented into Clinical Practice. Curr Allergy Asthma Rep. 2020 Jun 6;20(8):36. doi: 10.1007/s11882-020-00927-3.

[22] Gamble, A, Pham Q, Goyal S, Cafazzo JA. The Challenges of COVID-19 for People Living With Diabetes: Considerations for Digital Health. JMIR Diabetes. 2020 May 15;5(2):e19581. doi: 10.2196/19581.

[23] Boukhris M, Hillani A, Moroni F, Annabi MS, Addad F, Ribeiro MH, Mansour S, Zhao X, Ybarra LF, Abbate A, Vilca LM, Azzalini L. Cardiovascular Implications of the COVID-19 Pandemic: A Global Perspective. Can J Cardiol. 2020 May 16:S0828-282X(20)30464-5. doi: 10.1016/j.cjca.2020.05.018. Online ahead of print. 